\title{
Guía de manejo de la vía aérea en el paciente crítico adulto.
}

Artículo original: Higgs A, McGrath BA, Goddard C, Rangasami J, Suntharalingam G, Gale R, et al. Guidelines for the management of tracheal intubation in critically ill adults. Br J Anaesth. 2018;120(2):323-352. (

Monsalve Naharro JA (1), Canales Lara PM (1), Catalá Ripoll JV (2), Moreno Flores B (1)

(1) Complejo Hospitalario Universitario de Albacete.

(2) Hospital Francesc De Borja Gandía

\section{Resumen}

Se trata de una guía de práctica clínica de la Difficult Airway Society (DAS) británica que expone una estrategia para optimizar el manejo de la VA durante la intubación orotraqueal en el paciente crítico adulto, en cualquier localización hospitalaria.

\section{Introducción}

Se trata de una guía de práctica clínica de la Difficult Airway Society (DAS) británica que expone una estrategia para optimizar el manejo de la VA durante la intubación orotraqueal en el paciente crítico adulto, en cualquier localización hospitalaria.

El manejo de la vía aérea (VA) en el paciente crítico se realiza con frecuencia bajo una situación de urgencia. La probabilidad de que se presenten complicaciones y dificultades no previstas es mayor en las Unidades de Cuidados Intensivos (UCI) que en el quirófano (1). En este contexto, la vía aérea difícil (VAD) constituye un reto y su abordaje es una de las tareas de mayor exigencia al representar un riesgo vital.

\section{Guía clínica}

\section{Metodología}

La Difficult Airway Society británica creó un grupo de trabajo en 2014 con representación de la propia asociación, la Intensive Care Society (ICS), la Faculty of Intensive Care Medicine Joint Standards Committee (FICM) y la Royal College of Anaesthetists (RCoA). Llevaron a cabo una búsqueda bibliográfica en bases de datos (Medline, PubMed, Embase, Ovid y Google Scholar) mediante diversas palabras clave y filtros desde el año 2000 al 2014, que se repitieron periódicamente hasta mayo de 2017, recuperando artículos adicionales mediante referencias cruzadas y búsqueda manual. Se tuvieron en cuenta las opiniones de la comunidad de cuidados críticos con reuniones profesionales nacionales, un foro web y comentarios de las organizaciones profesionales pertinentes. Las recomendaciones fueron desarrolladas en función de la mejor evidencia disponible, suplementada por consenso de expertos en su ausencia.

\section{Factor humano}

Un elevado porcentaje de fracaso en el manejo de la vía aérea deriva de causas no técnicas, es decir, del factor humano, 
que incluye tanto las influencias ambientales como los comportamientos del equipo y el desempeño individual. Para su control se proporcionan una serie de ayudas cognitivas:

- Trabajo en equipo con líder, asignación de roles y colaboración efectiva (Figura 1).

- Listado de verificación o Checklist (Figura 2). Carro de vía aérea a la cabecera del paciente.

- Algoritmos de actuación (Figuras 3 y 4).

- Entrenamiento y simulación.

- Enfoque Vortex como estrategia para reducir la carga cognitiva y el error de fijación (2).

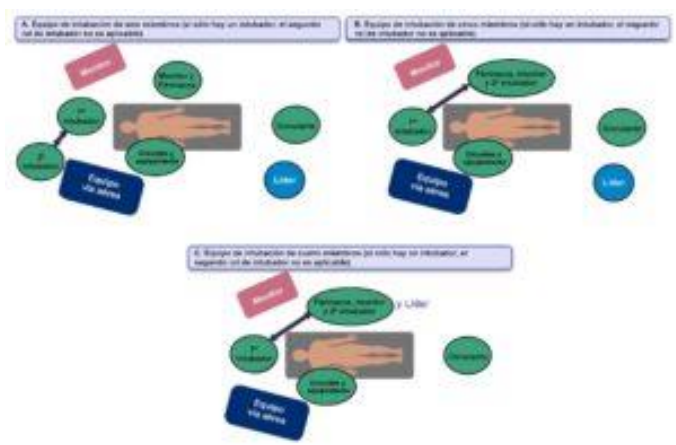

Figura 1. Composición y roles del equipo de intubación. Durante un procedimiento de intubación, los roles funcionales se pueden describir como: (1) primer intubador; (2) administrador de medicación (fármacos); (3) observador del estado clínico del paciente y monitores (monitor); (4) aplicador de presión cricoidea (cricoides); (5) asistente de equipo de vía aérea (equipo); (6) circulante para buscar equipo adicional o pedir ayuda; (7) segundo intubador; (8) líder de equipo coordinador (líder); y (9) estabilizador de columna cervical (rol específico para el trauma, que se debe agregar al complemento de cualquier equipo de intubación). Un miembro del equipo puede realizar más de un rol. La división detallada del trabajo dependerá de la cantidad de personal, en la figura se esquematizan 4 (A), 5 (B) y 6 (C) miembros. Además, los roles cambian después del primer intento fallido de intubación, cuando el segundo intubador se activa. Si el equipo consta sólo de un intubador, los roles permanecen sin cambios entre los intentos de intubación hasta la ayuda de expertos, si es que llega. El líder del equipo coordina el equipo con el intubador principal.

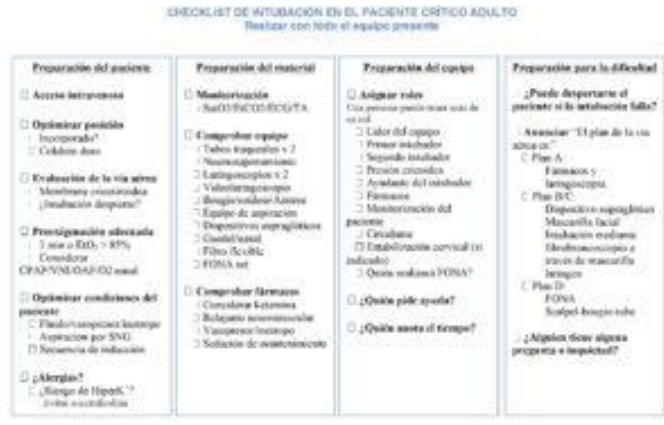

Figura 2. Listado de verificación de intubación. ET: end-tidal. CPAP: presión positiva continua en la vía aérea. VNI: ventilación no invasiva. OAF: oxígenoterapia de alto flujo. SNG: sonda nasogástrica. HiperK: hiperpotasemia. FONA: front of neck access.

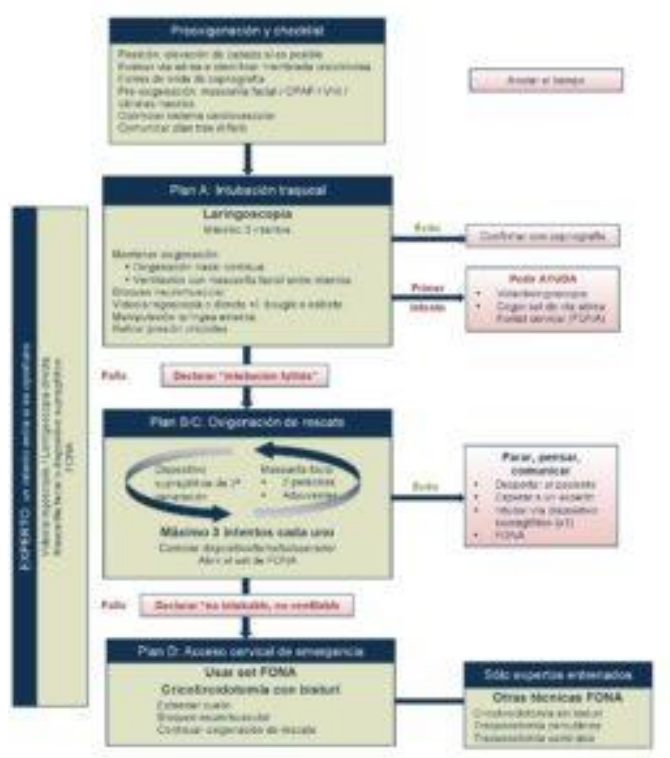

Figura 3. Algoritmo de intubación traqueal del paciente crítico adulto.

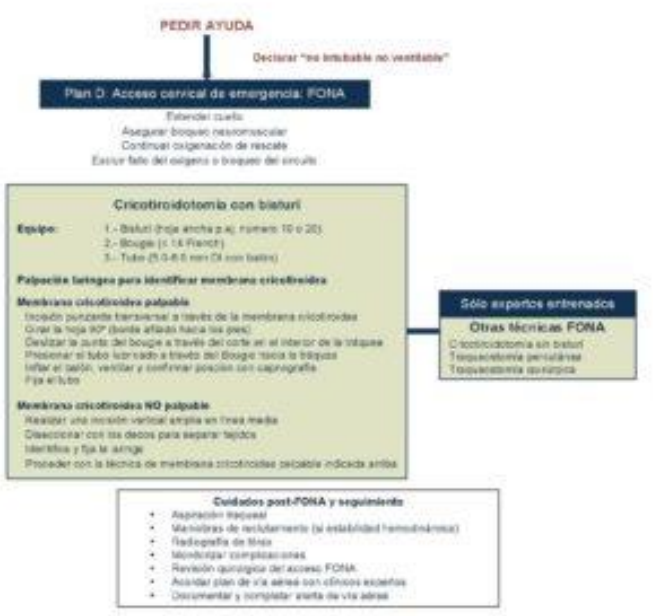

Figura 4. Algoritmo de intubación traqueal del paciente crítico adulto en situación no intubable no ventilable. 


\section{Evaluación}

Es necesario identificar el riesgo de aspiración y la dificultad en la intubación y en las técnicas de rescate. Se destacan la escala MACOCHA (Tabla 1) y la secuencia de palpación laríngea para identificar la membrana cricotiroidea.

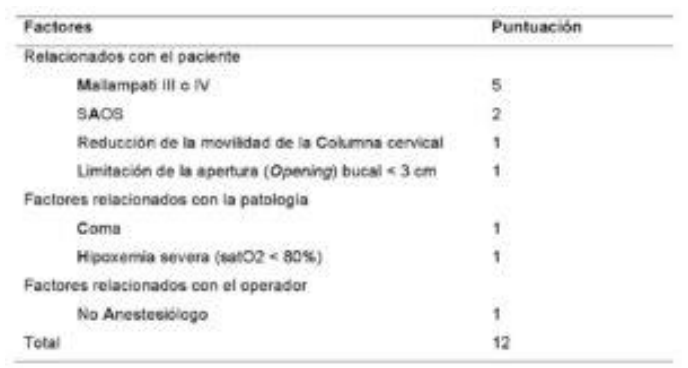

Tabla 1. MACOCHA score. Puntuación de 0 (fácil) a 12 (muy difícil).

Plan A: preparación, oxigenación, inducción, ventilación con mascarilla, intubación

- Listado de verificación y monitorización, incluyendo el tiempo.

- Solicitar ayuda si VAD prevista o tras intento fallido de intubación.

- Optimizar posición: sedestación; incorporación de cabeza 25$30 \%$; cabeza y cuello "en olfateo"; antitrendelemburg $30^{\circ}$ (sospecha de lesión de columna cervical); "en rampa" (paciente obeso).

- Preoxigenación: máscara facial con ajuste hermético y circuito con CPAP, BiPAP o gafas nasales de alto flujo (GNAF).

- Inducción y relajación neuromuscular. Evitar relajantes se asocia con más dificultad de intubación y de complicaciones.

- Inducción de secuencia rápida "modificada" si riesgo de broncoaspiración, con sonda nasogástrica con aspiración $\mathrm{y}$ con presión cricoidea (controvertida, evitar si dificulta ventilación o laringoscopia).

- Peroxigenación. Durante los intentos de intubación mediante cánulas nasales o bucal a 15 litros por minuto o con GNAF; entre intentos de intubación mediante ventilación con mascarilla facial y CPAP en pacientes con riesgo de hipoxia o si la hipercapnia puede ser problemática (acidosis metabólica, hipertensión intracraneal o pulmonar).

- Tras un máximo de 3 intentos debe declararse explícitamente la situación de intubación fallida (4 si la realiza un compañero más experimentado). Un intento se considera cuando la pala atraviesa la cavidad oral. Un intento fallido: asegurar disponibilidad de set de acceso frontal cervical de emergencia a la vía aérea (front of neck acces o FONA) y pedir ayuda.

- Deben considerarse como una opción para cualquier intubación, especialmente si se predice dificultad en la laringoscopia o si en el primer intento hay una pobre visión glótica con el laringoscopio.

- Confirmación de la intubación con capnografía.

- Maniobras de reclutamiento post-intubación.

Plan B/C: rescate de oxigenación usando dispositivo supraglótico o mascarilla facial tras intubación fallida

- Rescate de ventilación con mascarilla facial o con un dispositivo supraglótico (preferible) tras un intento fallido de intubación. Tras un máximo de 3 intentos declarar ventilación fallida. Un intento 
incluye cambiar el tamaño o el dispositivo supraglótico, o en caso de mascarilla facial cambiar tamaño, posición, operador, o necesidad de cánulas. El bloqueo neuromuscular mejora la ventilación.

- Este rescate implica que el equipo debe estar preparado para el FONA, cuyo set debe abrirse tras el primer intento de ventilación con mascarilla facial o dispositivo supraglótico.

- Si la ventilación es adecuada: para, piensa y comunica. Las opciones son: despertar al paciente (raramente aplicable al paciente crítico); esperar ayuda por un experto; considerar intubación a través del dispositivo supraglótico mediante fibrobroncoscopia; proceder al FONA.

- Si la ventilación es inadecuada: revierte las causas cuando sea posible y declara transición al escenario FONA en $<60$ segundos.

Plan D: FONA de emergencia

- Debe garantizarse: la administración de oxígeno al $100 \%$ por vía superior (mascarilla facial, dispositivo supraglótico, cánulas nasales), la posición del paciente con cuello extendido, y un adecuado bloqueo neuromuscular.

- Cricotiroidotomía mediante técnica de bisturí e introductor (Figura 4).

\section{Comentario}

Desde que en 1993 la Sociedad Americana de Anestesiólogos (ASA) publicara sus guías de manejo de la VAD, se han desarrollado múltiples algoritmos y guías de actuación a cargo de diversas sociedades internacionales de anestesiología. La naturaleza del manejo de la VAD no permite comparar unas guías sobre otras, a pesar de que sus recomendaciones se basan en análisis bibliográficos rigurosos y en la opinión de expertos.

El rendimiento de estas guías clínicas de vía aérea es desconocida en el paciente crítico (3) y pueden no ser útiles debido a que contemplan alternativas como despertar al paciente que no se pueden aplicar en una situación de emergencia. Por otro lado, es llamativo que aunque existen diferentes algoritmos de VAD adaptadados a las particularidades de los pacientes, como pediatría, obstetricia, cirugía torácica, o paciente obeso, no sea tan extendido el uso de guías en el paciente crítico. De hecho, aunque se habían desarrollado algunas aproximaciones como ayudas cognitivas (2), protocolos de intubación (3), o algoritmos (4), sólo se había publicado una guía clínica de intubación en el paciente crítico (5).

\section{Factor humano}

En situaciones de crisis, el abordaje de la vía aérea requiere de habilidades técnicas, pero también de las no técnicas. De hecho, un elevado porcentaje de fracaso deriva del factor humano. Por ello enfatiza la comunicación, el liderazgo y el trabajo en equipo, incluso por encima de la introducción de nuevos dispositivos o mejoras técnicas. Ayudas cognitivas como el "Enfoque Vortex" (2), suponen un recurso complementario para facilitar la implementación de las directrices de gestión y mejorar el rendimiento de los equipos clínicos disminuyendo los errores durante una emergencia.

Es incuestionable que es preciso una adecuada preparación del personal, equipo, material, medicación, plan de 
rescate, asignación de roles y estrategia de intubación. Aunque la guía incluye un listado de verificación previo a la intubación, un reciente estudio multicéntrico no objetivó que el uso de un Check list escrito mejorara el número de complicaciones con respecto a la preparación habitual verbal (6).

\section{Valoración}

Ninguno de los múltiples factores predictivos de VAD es totalmente fiable. La única herramienta validada en el paciente crítico es la escala MACOCHA. En cuanto a la ecografía cervical se destaca como técnica más precisa que la palpación laríngea para identificar la membrana cricotiroidea, que valora además tamaño, profundidad, vasos y tejido tiroideo, por lo que puede ser útil si el tiempo lo permite.

Plan A: preparación, oxigenación, inducción, ventilación con mascarilla, intubación

Es prioritaria la oxigenación, que incluye tanto la pre como la peroxigenación, con un abordaje modificado de la intubación de secuencia rápida. El uso de dispositivos que proporcionen presión positiva continua ha demostrado una menor hipoxemia durante la intubación en pacientes con insuficiencia respiratoria, ofreciendo ventajas frente a los modelos clásicos de preoxigenación.

Se propone un uso precoz de vídeolaringoscopios, que ya se describen como una alternativa superior a la laringoscopia directa tanto para el tratamiento primario de la VAD como para el rescate tras el fracaso de la laringoscopia directa. El grado de evidencia de estas afirmaciones es bueno en el ámbito de la anestesiología (7). Sin embargo, estos resultados no se han extrapolado al paciente crítico (8), donde los datos publicados son generalmente de poca calidad y con limitada evidencia, incluso a veces omitiéndose el grado de entrenamiento. En manos poco experimentadas, consiguen enlentecer el procedimiento $\mathrm{y}$, en pacientes críticos con pocas reservas, unos segundos adicionales pueden tener consecuencias fatales.

Con respecto al uso de relajantes neuromusculares, la guía establece un punto y aparte a otras recomendaciones previas de administración sólo si se verifica la posibilidad de ventilar al menos con mascarilla facial. Ya tenemos evidencia de que su uso mejora las condiciones de intubación y de ventilación reduciendo el número de intentos y las complicaciones añadidas $(1,9)$.

Muchas catástrofes en vía aérea ocurren tras la intubación, por lo que esta guía enfatiza métodos para evitar sus complicaciones. En este contexto se recomienda que la comprobación de la intubación se realice de forma mandatoria mediante capnografía, como también recomiendan guías de reanimación cardiopulmonar y otras guías de VAD (10).

Plan B/C: rescate de oxigenación usando dispositivo supraglótico o mascarilla facial tras intubación fallida

El plan secuencial a usar cuando falla la intubación traqueal debe priorizar la oxigenación y reducir la posibilidad de trauma sobre la vía aérea con los intentos repetidos de inserción de dispositivos. El algoritmo combina los Planes B y $\mathrm{C}$ a diferencia de guías anteriores (10), debido a que, en la práctica, la distinción entre oxigenar a través de ventilación con mascarilla facial o a través de un dispositivo supraglótico es conceptual, y el rescate de la oxigenación puede alternar ambos 
dispositivos de ventilación, como se reconoce en el modelo del Vórtex.

\section{Plan D: FONA de emergencia}

Con respecto a la situación no intubable no ventilable, se mantiene la recomendación del FONA de la guía previa de VAD de la DAS (10), acelerando la toma de decisión sin esperar a la cifra baja de saturación de oxígeno como gatillante, y mediante el acceso quirúrgico con un bisturí y un introductor. No existe acuerdo en cuanto a la técnica de entre otras guías de VAD. En ésta se basan en los criterios de un procedimiento ideal, en reducir alternativas (que ha demostrado un aumento significativo en el tiempo de reacción) y en las limitaciones de otras prácticas. No obstante, se reconoce que la evidencia para recomendarla es limitada y no excluye las técnicas por punción. El procedimiento a elegir debe ser aquél con el que se tenga mayor disponibilidad y experiencia. Lo más importante es el entrenamiento para hacerlo eficaz y seguro.

Para finalizar queremos destacar que, como cualquier guía clínica, su intención radica en constituir un estándar mínimo en la práctica clínica, sin que sea un sustituto absoluto de un correcto juicio clínico, que debe adaptarse a las competencias específicas del profesional, la disponibilidad de material, y a las características particulares de cada paciente. En cualquier caso, supone un avance importante, debido a que la implementación de paquetes de medidas o protocolos de intubación en UCI puede contribuir a reducir de forma significativa las complicaciones severas inmediatas asociadas a dicho procedimiento (3).

\section{Bibliografía}

1.- Cook TM, Woodall N, Frerk C; Fourth National Audit Project. Major complications of airway management in the UK: results of the Fourth National Audit Project of the Royal College of Anaesthetists and the Difficult Airway Society. Part 1: anaesthesia. $\mathrm{Br} \mathrm{J}$ Anaesth. 2011;106(5):617-31. (PubMed) (HTML)

2.- Chrimes N. The Vortex: a universal 'highacuity implementation tool' for emergency airway management. Br J Anaesth. 2016;117 Suppl 1:i20-i27. (ubMed) (ㅍML)

3.- Jaber S, Jung B, Corne P, Sebbane M, Muller L, Chanques G, et al. An intervention to decrease complications related to endotracheal intubation in the intensive care unit: a prospective, multiple-center study. Intensive Care Med. 2010;36(2):248-55. (PubMed)

4.- Walz JM, Zayaruzny M, Heard SO. Airway management in critical illness. Chest. 2007;131(2):608-20. (PubMed)

5.- Myatra SN, Ahmed SM, Kundra P, Garg R, Ramkumar V, Patwa A, et al. Republication: All India Difficult Airway Association 2016 Guidelines for Tracheal Intubation in the Intensive Care Unit. Indian J Crit Care Med.

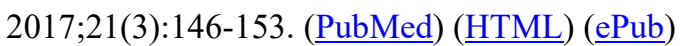

6.- Janz DR, Semler MW, Joffe AM, Casey JD, Lentz RJ, deBoisblanc BP, et al. A Multicenter Randomized Trial of a Checklist for Endotracheal Intubation of Critically Ill Adults. Chest. 2018;153(4):816-824. (ubMed)

7.- Lewis SR, Butler AR, Parker J, Cook TM, Schofield-Robinson OJ, Smith AF. Videolaryngoscopy versus direct laryngoscopy for adult patients requiring tracheal intubation: a Cochrane Systematic Review. Br J Anaesth. 2017;119(3):369-383. (PubMed)

8.- Jiang J, Ma D, Li B, Yue Y, Xue F. Video laryngoscopy does not improve the intubation outcomes in emergency and critical patients - a systematic review and meta-analysis of randomized controlled trials. Crit Care. 2017;21(1):288. (ubMed) (HTML1) ( (PDF)

9.- Lundstrøm LH, Duez CHV, Nørskov AK, Rosenstock CV, Thomsen JL, Møller AM, et al. Effects of avoidance or use of neuromuscular blocking agents on outcomes in tracheal 
intubation: a Cochrane systematic review. Br J Anaesth. 2018;120(6):1381-1393. (PubMed)

10.- Frerk C, Mitchell VS, McNarry AF, Mendonca C, Bhagrath $\mathrm{R}$, Patel A, et al. Difficult Airway Society 2015 guidelines for management of unanticipated difficult intubation in adults. $\mathrm{Br} \mathrm{J}$ Anaesth.

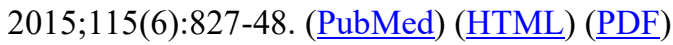

Correspondencia al autor

Jose Ángel Monsalve Naharro

jose_mn@hotmail.com

Facultativo Especialista de Área.

Servicio de Anestesiología, Reanimación y Terapéutica del Dolor.

Complejo Hospitalario Universitario de Albacete.

Aceptado para el blog en abril de 2019. 